\title{
Optical coherence tomography of actinic keratoses and basal cell carcinomas - differentiation by quantification of signal intensity and layer thickness
}

\author{
S. Schuh, ${ }^{1}$ R. Kaestle, ${ }^{1}$ E. C. Sattler, ${ }^{2, a}$, J. Welzel ${ }^{1, a, *}$ \\ ${ }^{1}$ Department of Dermatology and Allergology, General Hospital Augsburg, Augsburg, Germany \\ ${ }^{2}$ Department of Dermatology and Allergology, Ludwig-Maximilian University of Munich, Munich, Germany \\ *Correspondence: J. Welzel. E-mail: julia.welzel@klinikum-augsburg.de
}

\begin{abstract}
Background Previous studies have shown that actinic keratoses (AKs) and basal cell carcinomas (BCCs) can be diagnosed by optical coherence tomography (OCT) based on morphological characteristics. There is a lack of systematic studies that give standardized information on signal intensity and layer thickness of AKs and BCCs.

Objective The aim of this study was to find out if AKs and BCCs can be objectively diagnosed through standardized measurement of signal intensity and layer thickness and to use OCT as a non-invasive objective method for the diagnosis and evaluation of AKs and BCCs. Additionally, tumour and skin layer thickness were investigated in correlation with histology.

Methods In this experimental study, 301 lesions (188 BCCs and 113 AKs) of 125 patients were clinically as well as dermoscopically diagnosed and investigated with OCT before therapy. Normal perilesional skin served as control.

Results It is possible to differentiate BCCs and AKs from normal skin in OCT due to the decrease of local signal intensity in affected skin layers in relation to adjacent healthy skin. In AKs, a strong thickness increase of the stratum corneum and epidermis compared to normal skin were observed. For the distinction between AKs and BCCs, a drop of signal intensity in the dermis of AKs towards BCCs and a thicker epidermis of AKs in contrast to BCCs were registered. All results are statistically highly significant $(P<0.0001)$. Besides, a strong correlation of tumour and skin layer thickness of BCCs and AKs in OCT with histology was found.

Conclusion Through standardized measurement of signal intensity and layer thickness, BCCs and AKs can be objectively diagnosed and distinguished from each other with OCT. This will further improve the use of OCT as a non-invasive objective method for the diagnosis and treatment monitoring of these diseases.
\end{abstract}

\section{Conflicts of interest}

None.

\section{Funding sources}

None.

\section{Introduction}

Nowadays, non-melanoma skin cancer (NMSC) like actinic keratosis (AKs) and basal cell carcinomas (BCCs) are usually diagnosed clinically with a sensitivity of 56-90\% and specificity of 75-90\%. ${ }^{1}$ However, superficial BCCs are not easy to diagnose because they can be mistaken for psoriatic plaques or Bowen's disease. ${ }^{2}$ The accuracy of diagnosing a BCC can be improved from $65.8 \%$ with physical examination to $76.2 \%$ by using

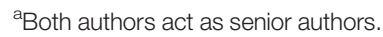

dermoscopy and to $87.4 \%$ if optical coherence tomography (OCT) is used additionally. ${ }^{3}$

Prior studies already defined morphologic criteria for the diagnosis of BCCs and AKs in OCT. ${ }^{4-6}$ Due to developments of the high-definition OCT (HD-OCT), more structural details are known to identify even subtypes of BCCs, and to discriminate AKs from SCCs and normal skin. ${ }^{7-10}$ Typically, there is no regular layering of the skin, especially of the often thickened epidermis (in AKs and BCCs). ${ }^{11}$ BCCs present as ovoid dark structures surrounded by fibrous stroma in the dermis. ${ }^{4,8,9,12}$ AKs show hyperreflective flakes and streaks in the upper epidermis, and 
dark bands in the thickened stratum corneum and epidermis. ${ }^{6,13}$ But, despite all these efforts, ${ }^{5,14}$ the differentiation between BCCs and AKs and other skin diseases remains difficult in some cases. This is why more objective non-invasive measurements of the skin like Gao et al. ${ }^{15}$ are needed.

Thus, the aim of this study was to objectively diagnose and discriminate AKs and BCCs through standardized measurement of signal intensity and layer thickness using OCT.

\section{Methods}

\section{Patients and study performance}

One hundred and twenty-five patients (51 female, 74 male, median age 70.5, range 39-95 years) with 301 lesions (113 AKs and 188 BCCs) were recruited in an experimental study. Approvement was obtained by the Ethical Committee of the Ludwig-Maximilian University (Project number 221-11) and by the ethic vote of the University of Lübeck (1997). The study was conducted from September 2011 to March 2014 at the Department of Dermatology and Allergology of the General Hospital in Augsburg. The principles of the declaration of Helsinki and international guidelines concerning human studies were adhered to at any time.

Inclusion criteria were age over 18 years, good quality OCT images, unequivocal clinical and dermoscopic diagnosis of AKs/ BCCs and unaffected perilesional skin as a control site. After written informed consent, the patients were clinically and dermoscopically examined and the diagnosis was established. The NMSC was photographed with a Panasonic Lumix DMC-TZ8. Every lesion and normal perilesional skin as a control were measured with OCT before therapy. If the NMSC was larger than $6 \mathrm{~mm}$, the middle of the lesion was scanned. One hundred and twenty lesions (97 BCCs, 23 AKs) were surgically removed and histopathologically confirmed by a certified dermatopathologist. One hundred and eighty-one (91 BCCs, 90 AKs) skin changes received topical treatment.

\section{Optical coherence tomography}

The OCT (VivoSight ${ }^{\circledR}$; Michelson Diagnostics, Kent, UK) scans an area of $6 \times 6 \mathrm{~mm}^{2}$, reaching a penetration depth of 1.5 to $2 \mathrm{~mm}$ with $<7.5 \mu \mathrm{m}$ as a lateral and $5-10 \mu \mathrm{m}$ as an axial resolution. ${ }^{16}$ This multibeam swept source Fourier domain OCT uses an on Michelson interferometry based laser (HSL 2000; Santec Corporation, Komaki, Japan) with a wavelength of $1305 \mathrm{~nm} .{ }^{12,16}$ Neither oil nor ultrasonic gel are needed.

\section{Image analysis}

Only OCT images of clinically and dermoscopically unequivocal or histopathologically confirmed lesions were taken in vivo, in real-time as sequences of video material, and were saved for the analysis with the software Image $\mathrm{J}$ as single images. In order not to influence the signal intensity measurements, the raw images were analysed, with no image adjustments or enhancements applied beforehand.

The mean OCT signal intensity in each image was measured at the same depths (BCC tumour/adjacent dermis) for each lesion, by use of the Image J polygon and histogram tools. These tools allow the user to manually select an arbitrarily shaped polygon region of the image, which is then automatically processed to provide statistics of all of the enclosed pixels, including the mean grey level. A typical selected region approximately $0.8 \mathrm{~mm} \times 0.2 \mathrm{~mm}$ in extent contained over 12000 image pixels. This method provides a good estimate of the mean signal intensity in the selected region, which is not sensitive to speckle noise or minor local variations in intensity.

With Image J, the thickness of BCC tumours, the stratum corneum, the epidermis in AKs and the epidermis above BCCs were measured. The epidermis ranges from the skin surface reflection to the first signal intensity reduction, the dermal-epidermal junction (Fig. 1c). ${ }^{17}$ The stratum corneum can be observed in normal skin as a small band beneath the strong reflection (Fig. 1c). Thickness measurements in the OCT image and in histology were carried out from the top of the skin surface to the bottom of the tumour margin. The NMSC affected mainly the head and neck (103 AKs, 94 BCCs), trunk (3 AKs, 73 BCCs) and extremities (7 AKs, 21 BCCs), but not palmoplantar skin.

\section{Statistical analysis}

The data were collected with Microsoft ${ }^{\circledR}$ Excel $^{\circledR}$ for Mac 2011 and statistical calculations were conducted using IBM $^{\circledR}$ SPSS $^{\circledR}$ Statistics software for Mac (SPSS 21.0, IBM Corp., Armonk, NY, USA). A summary of the OCT results on NMSC is shown in Table 1 . With normally distributed data, paired sample t-tests were used for intraindividual comparisons of BCCs/AKs and healthy skin. When the data failed the normality test (like Kolmogorov-Smirnov Test or modified by Lilliefors), a Wilcoxon test was done. The Mann-Whitney $U$-Test for independent samples was used for an interindividual comparison of AKs with BCCs. Spearman's correlation coefficients $(r)$ were calculated to correlate OCT with histology. A $P$-value below 0.05 was considered as statistically significant.

\section{Results}

\section{Signal intensity of BCCs and AKs as well as skin layer thickness of AKs compared to perilesional normal skin visualized by OCT}

From 188 BCCs, the mean signal intensity of the BCC, the epidermis above the BCC as well as of the dermis beneath the BCC was determined in comparison to the perilesional normal skin layer. Attributable to the BCC, there is a decrease in the local signal intensity in all affected skin layers in relation to adjacent healthy skin (Fig. 1a). Descriptive analysis for BCCs and AKs can be seen in Table 2a. 
(a) Signal intensity of BCCs
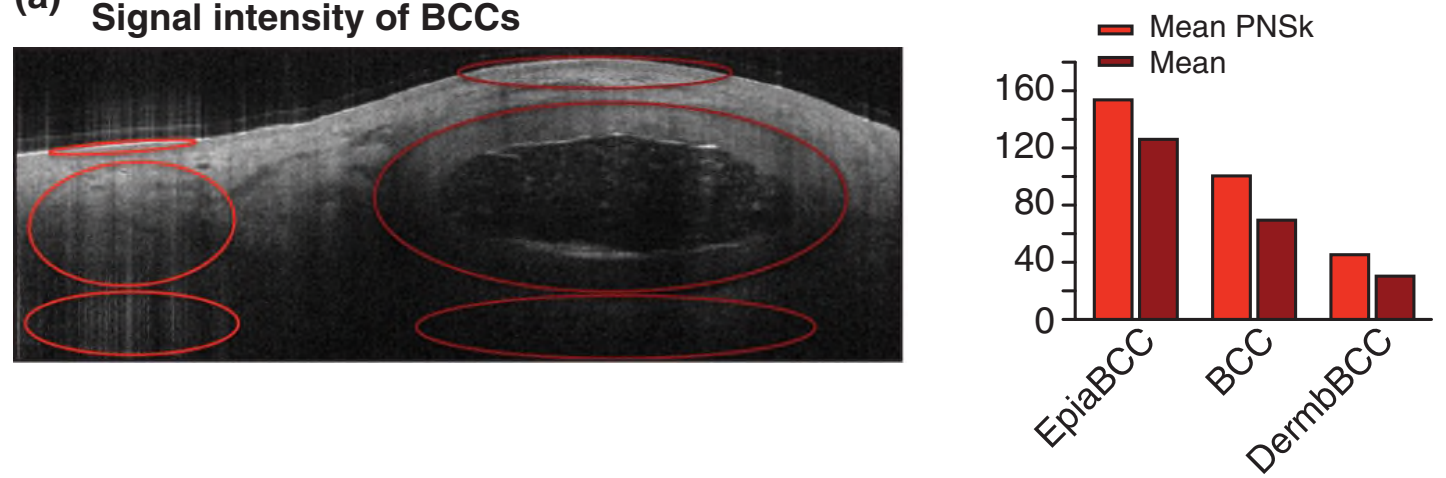

(b)

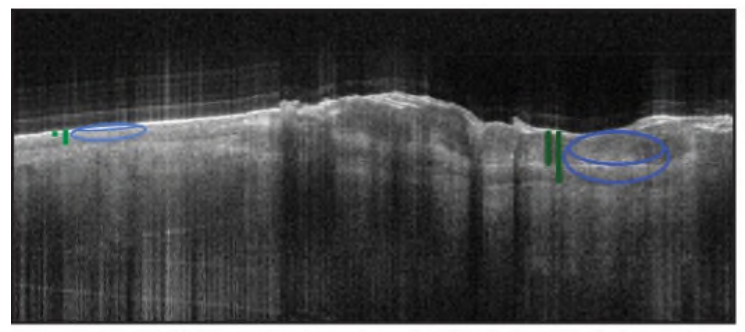

(d) Mean epidermis thickness
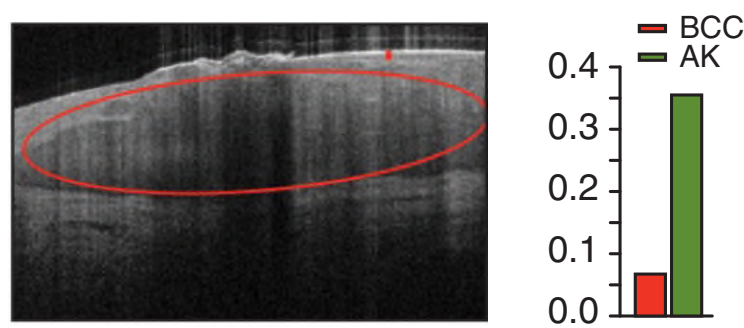

Signal intensity of AKs

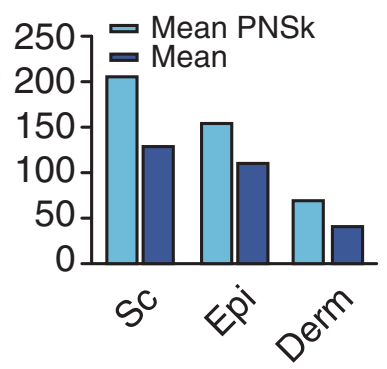

(c)

\section{Thickness of AKs}

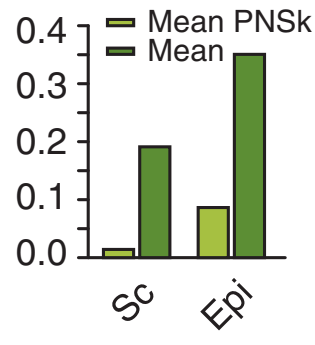

(e) Mean signal intensity of BCC/adjacent dermis of AK
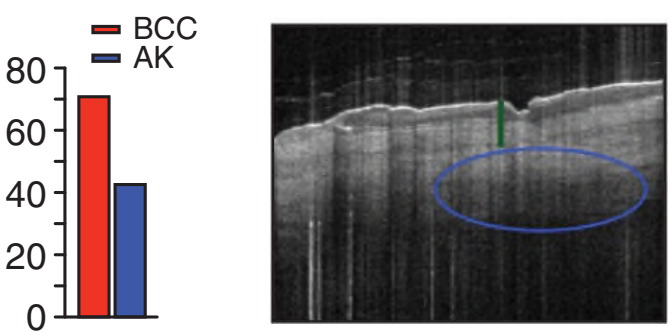

Figure 1 (a) Signal intensity of BCCs and (b) AKs as well as (c) skin layer thickness of AKs compared to perilesional normal skin (PNSk) visualized by OCT; Distinction between AKs and BCCs illustrated by OCT through (d) epidermis thickness and (e) signal intensity measurement.

Table 1 Summary of the OCT results on NMSC concerning signal intensity $(\mathrm{SI})$ and layer thickness $(\mathrm{T})$; the symbol before the slash refers to the first tumour entity and the symbol after the slash to the second tumour entity or PNSk

\begin{tabular}{llllll} 
& SI Sc & SI Epi & SI Derm & T Sc & T Epi \\
\hline AK/PNSk & $-/ 0$ & $-/ 0$ & $-/ 0$ & $++/ 0$ & $++/ 0$ \\
BCC/PNSk & & $-/ 0$ & $-/ 0$ & & $-/ 0$ \\
AK/BCC & & & $-/-$ & & $++/-$
\end{tabular}

++ very high, + high, o normal, - low, - very low

Epi, epidermis; Derm, dermis; PNSk, perilesional normal skin; Sc, stratum corneum.
Significant differences between the signal intensity of the epidermis above the BCC, the BCC itself, as well as below the BCC and normal skin were observed $(P=0.000)$.

One hundred and thirteen AKs were measured concerning mean thickness and signal intensity of the stratum corneum and of the epidermis, and the signal intensity of the dermis each time compared to perilesional healthy skin. A decline of signal intensity in afflicted skin layers as compared with adjacent normal skin is visible (Fig. 1b). But, a strong increase in the thickness of the stratum corneum and epidermis towards healthy skin can be registered (Fig. 1c). 
Table 2 Statistical analysis of (a) BCCs and AKs in comparison to perilesional normal skin measured by OCT; (b) the differentiation between BCCs and AKs measured by OCT; (c) signal intensity and skin layer thickness of the histologically confirmed subgroup in contrast to normal skin, (d) the distinction between histologically confirmed AKs and BCCs

\begin{tabular}{|c|c|c|c|c|c|c|c|c|c|c|c|}
\hline \multirow[t]{2}{*}{ (a) } & & \multicolumn{5}{|c|}{ Signal intensity } & \multicolumn{5}{|l|}{ Thickness } \\
\hline & & Mean PNSk & Mean & SD PNSk & SD & $P$-value & Mean PNSk & Mean & SD PNSk & SD & $P$-value \\
\hline \multirow[t]{3}{*}{ AK } & Sc & 207.4 & 131.2 & 21.8 & 23.5 & $<0.0001$ & 0.017 & 0.193 & 0.006 & 0.119 & $<0.0001$ \\
\hline & Epi & 154.2 & 111.2 & 15.8 & 20.7 & $<0.0001$ & 0.089 & 0.355 & 0.025 & 0.181 & $<0.0001$ \\
\hline & Derm & 70.9 & 42.9 & 23.9 & 18.2 & $<0.0001$ & & & & & \\
\hline \multirow[t]{3}{*}{$\mathrm{BCC}$} & EpiaBCC & 155.5 & 129.5 & 15.0 & 19.1 & $<0.0001$ & & & & & \\
\hline & $\mathrm{BCC}$ & 103.4 & 71.5 & 18.9 & 21.0 & $<0.0001$ & & & & & \\
\hline & DermbBCC & 45.7 & 31.2 & 14.7 & 14.5 & $<0.0001$ & & & & & \\
\hline
\end{tabular}

\begin{tabular}{|c|c|c|c|c|c|c|}
\hline \multirow[t]{2}{*}{ (b) } & \multicolumn{3}{|c|}{ Signal intensity of $\mathrm{BCC} /$ dermis of $\mathrm{AK}$} & \multicolumn{3}{|c|}{ Epidermis thickness } \\
\hline & Mean & SD & $P$-value & Mean & SD & $P$-value \\
\hline BCC & 71.5 & 21.0 & $<0.0001$ & 0.065 & 0.015 & $<0.0001$ \\
\hline AK & 42.9 & 18.2 & & 0.355 & 0.181 & \\
\hline
\end{tabular}

\begin{tabular}{|c|c|c|c|c|c|c|}
\hline \multirow[t]{2}{*}{ (c) } & & \multicolumn{5}{|c|}{ Signal intensity } \\
\hline & & Mean PNSk & Mean & SD PNSk & SD & $P$-value \\
\hline \multirow[t]{3}{*}{ AK } & Sc & 206.5 & 124.6 & 21.5 & 23.9 & $<0.0001$ \\
\hline & Epi & 153.8 & 105.1 & 15.7 & 20.3 & $<0.000$ \\
\hline & Derm & 59.0 & 35.6 & 16.8 & 11.8 & $<0.000$ \\
\hline \multirow[t]{3}{*}{$\mathrm{BCC}$} & EpiaBCC & 153.3 & 126.8 & 14.5 & 19.2 & $<0.000$ \\
\hline & BCC & 100.2 & 66.9 & 20.4 & 21.8 & $<0.000$ \\
\hline & DermbBCC & 44.4 & 29.7 & 14.4 & 13.7 & $<0.000$ \\
\hline
\end{tabular}

\begin{tabular}{|c|c|c|c|c|c|c|}
\hline \multirow[t]{2}{*}{ (d) } & \multicolumn{3}{|c|}{ Signal intensity of $\mathrm{BCC} /$ dermis of $\mathrm{AK}$} & \multicolumn{3}{|c|}{ Epidermis thickness } \\
\hline & Mean & SD & $P$-value & Mean & SD & $P$-value \\
\hline $\mathrm{BCC}$ & 66.9 & 21.8 & $<0.0001$ & 0.068 & 0.015 & $<0.0001$ \\
\hline AK & 35.6 & 11.8 & & 0.397 & 0.172 & \\
\hline
\end{tabular}

DermbBCC, dermis beneath BCC; Derm, dermis; EpiaBCC, epidermis above BCC; Epi, epidermis; PNSk, perilesional normal skin; Sc, stratum corneum; SD, standard deviation; signal intensity in greyscale $(0=$ black, $255=$ white $)$, thickness in $\mathrm{mm}, P$-value in bold letters.

Statistically significant distinctions were found between AKs and normal skin regarding the thickness of the stratum corneum, the epidermis and all three cases of signal intensity comparisons $(P=0.000)$.

\section{Distinction between AKs and BCCs in OCT}

The mean signal intensity of BCCs is brighter compared to the mean signal intensity of the dermis of AKs (Fig. 1e). AKs show a higher mean epidermis thickness in comparison to the epidermis above BCCs (Fig. 1d).

Therefore, AKs can be significantly discriminated from BCCs through epidermis thickness and signal intensity in the OCT (both $P=0.000$ see Table $2 b$ ).

\section{Comparisons of OCT with histology}

The histologically confirmed subgroup of 88 patients ( 32 female, 56 male, median age of 70.45, range 39-95) with 97 BCCs and 23 AKs provides the same results as the entire group (Table $2 \mathrm{c}$ and $\mathrm{d}$ ). Table $3 \mathrm{a}$ shows the strong correlation of tumour and skin layer thickness measurement of BCCs and AKs using OCT with histology. With three BCCs, no histological measurements were possible, leaving 94 BCCs for correlation, including BCCs larger than the $2 \mathrm{~mm}$ penetration of OCT (Table $3 \mathrm{~b}$ ). When comparing the signal intensities of histological BCC subtypes, superficial BCCs showed higher mean signal intensity (87.5) than BCCs of sclerosing (59.4) or nodular subtype (57.7).

\section{Discussion}

Our intention was to diagnose and differentiate BCCs and AKs based on quantifiable objective parameters, e.g. the signal intensity of BCCs is low compared to adjacent healthy skin. Even, Greaves et al. ${ }^{18,19}$ report that the mean grey value is a quantitative method of characterizing tissue due to the correlation of mean grey values with fibrosis, which was proven by histology and other ex vivo analysis. Furthermore, we detected that AKs have a statistically lower signal intensity in all examined skin lay- 
Table 3 (a) Statistical results of the tumour and skin layer thickness correlation of BCCs and AKs with histology; Sc, stratum corneum; Epi, epidermis; $r$, Spearman's correlation coefficient; the correlation is significant at a level of 0.01 (both sides); (b) point cloud as a graphic display of the correlation of BCC tumour thickness measurement using OCT with histology; thickness in $\mathrm{mm}$; in this point cloud $2 \mathrm{~mm}$ include all tumours $>2 \mathrm{~mm}$

\begin{tabular}{lllll} 
(a) & & \multicolumn{2}{l}{ Correlation OCT/histology } \\
\cline { 3 - 5 } & & Spearman's rho & $\begin{array}{l}\boldsymbol{P} \text {-value of } \\
\text { correlation }\end{array}$ & $\begin{array}{l}\boldsymbol{P} \text {-value of } \\
\text { Wilcoxon tests }\end{array}$ \\
\hline AK & Sc & $r=0.894$ & $<0.0001$ & 0.013 \\
& Epi & $r=0.951$ & $<0.0001$ & 0.005 \\
BCC & & $r=0.879$ & $<0.0001$ & $<0.0001$ \\
\hline
\end{tabular}

(b)

$\mathrm{BCC}$ tumor thickness correlation

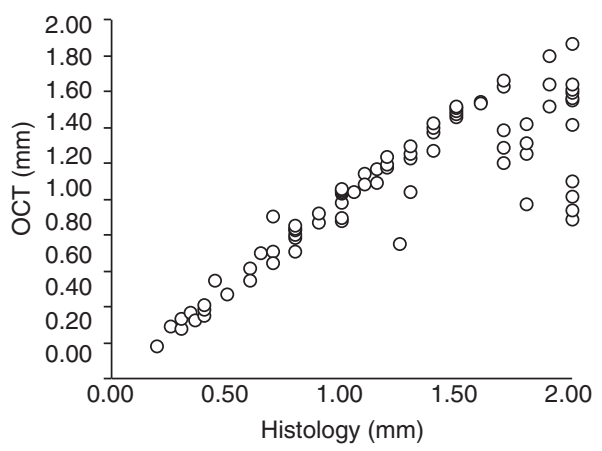

ers than perilesional normal skin despite the findings in Barton et al. ${ }^{6}$ Therein, a severely thickened stratum corneum was stated as a typical characteristic for AKs in OCT, which we can confirm, besides a strong increase in the epidermal thickness in AKs towards healthy skin. ${ }^{6}$ Since AKs and BCCs can often not be discriminated from each other, a difference in the signal intensity as well as in the epidermal thickness was found. ${ }^{5,14}$ Further studies could rule out differential diagnoses by signal intensity measurement.

As only 120 lesions were confirmed histologically, which showed the same results as the whole group, there is no absolute certainty that the other 181 lesions are correctly identified. Due to the maximum penetration of $2 \mathrm{~mm}$ in OCT, tumours $>2 \mathrm{~mm}$ could not be completely measured. Moreover, tumours with less than $1 \mathrm{~mm}$ thickness in OCT correlated well with histology. This is of high clinical relevance, because the cut-off for good treatment effects with imiquimod and photodynamic therapy is $1 \mathrm{~mm} .^{20}$ Differences in the signal intensity and layer thickness regarding the whole lesion are possible due to the choice of single images and because of interobserver variability. However, the control group is dependent on each patient and there is no normal healthy skin as a reference in general.

Conclusively, our results show that signal intensity and layer thickness are diagnostically useful and valid parameters, which might serve to further improve sensitivity and specificity of OCT diagnosis purely on image morphology. Therefore, these image analysis tools should be added to the software on commercially available OCT equipment.

\section{Acknowledgements}

Many thanks to Dipl.-Math. Karlheinz Haude for statistical counselling.

\section{References}

1 Mogensen M, Jemec GB. Diagnosis of nonmelanoma skin cancer/keratinocyte carcinoma: a review of diagnostic accuracy of nonmelanoma skin cancer diagnostic tests and technologies. Dermatol Surg 2007; 33: 1158-1174.

2 Madan V, Lear JT, Szeimies R. Non-melanoma skin cancer. Lancet 2010; 375: 673-685.

3 Ulrich M, von Braunmuehl T, Kurzen $\mathrm{H}$ et al. The sensitivity and specificity of optical coherence tomography for the assisted diagnosis of nonpigmented basal cell carcinoma: an observational study. Br J Dermatol 2015; 173: 428-435.

4 Gambichler T, Orlikov A, Vasa R et al. In vivo optical coherence tomography of basal cell carcinoma. J Dermatol Sci 2007; 45: 167-173.

5 Mogensen M, Jørgensen TM, Nürnberg BM et al. Assessment of optical coherence tomography imaging in the diagnosis of non-melanoma skin cancer and benign lesions versus normal skin: observer-blinded evaluation by dermatologists and pathologists. Dermatol Surg 2009; 35: 965972.

6 Barton JK, Gossage KW, Xu W et al. Investigating sun-damaged skin and actinic keratosis with optical coherence tomography: a pilot study. Technol Cancer Res Treat 2003; 2: 525-535.

7 Boone MA, Marneffe A, Suppa M et al. High-definition optical coherence tomography algorithm for the discrimination of actinic keratosis from normal skin and from squamous cell carcinoma. J Eur Acad Dermatol Venereol 2015; 29: 1606-1615.

8 Boone MA, Norrenberg S, Jemec GB, Del Marmol V. Imaging of basal cell carcinoma by high-definition optical coherence tomography: histomorphological correlation. A pilot study. Br J Dermatol 2012; 167: 856864.

9 Boone MA, Suppa M, Pellacani G et al. High-definition optical coherence tomography algorithm for discrimination of basal cell carcinoma from clinical BCC imitators and differentiation between common subtypes. $J$ Eur Acad Dermatol Venereol 2015; 29: 1771-1780.

10 Maier T, Braun-Falco M, Laubender RP et al. Actinic keratosis in the enface and slice imaging mode of high-definition optical coherence tomography and comparison with histology. Br J Dermatol 2013; 168: 120-128.

11 Themstrup L, Banzhaf CA, Mogensen M, Jemec GB. Optical coherence tomography imaging of non-melanoma skin cancer undergoing photodynamic therapy reveals subclinical residual lesions. Photodiagnosis Photodyn Ther 2014; 11: 7-12.

12 Welzel J. Optical coherence tomography in dermatology: a review. Skin Res Technol 2001; 7: 1-9.

13 Korde VR, Bonnema GT, Xu W et al. Using optical coherence tomography to evaluate skin sun damage and precancer. Lasers Surg Med 2007; 39: 687-695.

14 Jørgensen TM, Tycho A, Mogensen M et al. Machine-learning classification of non-melanoma skin cancers from image features obtained by optical coherence tomography. Skin Res Technol 2008; 14: 364-369.

15 Gao W, Zakharov VP, Myakinin OO et al. Medical images classification for skin cancer using quantitative image features with optical coherence tomography. J Innov Opt Health Sci 2016; 9: 1650003-11650003-9.

16 Michelson Diagnostics. VivoSight OCT Scanner- Technical Specification. Available at: http://www.vivosight.com/wp-content/uploads/2013/02/ 
1003.SP_.638-Issue-1-VivoSight-Technical-Specification.pdf (last accessed: June 2015).

17 Gambichler T, Matip R, Moussa G et al. In vivo data of epidermal thickness evaluated by optical coherence tomography: effects of age, gender, skin type, and anatomic site. J Dermatol Sci 2006; 44: 145-152.

18 Greaves NS, Benatar B, Whiteside S et al. Optical coherence tomography: a reliable alternative to invasive histological assessment of acute wound healing in human skin? Br J Dermatol 2014; 170: 840-850.
19 Greaves NS, Iqbal SA, Hodgkinson T et al. Skin substitute-assisted repair shows reduced dermal fibrosis in acute human wounds validated simultaneously by histology and optical coherence tomography. Wound Repair Regen 2015; 23: 483-494.

20 Christensen E, Mørk C, Skogvoll E. High and sustained efficacy after two sessions of topical 5-aminolaevulinic acid photodynamic therapy for basal cell carcinoma: a prospective, clinical and histological 10-year follow-up study. Br J Dermatol 2012; 166: 1342-1348. 